\title{
A topological version of Bertini's theorem
}

\author{
by ARTuR PiȨKosz (Kraków)
}

\begin{abstract}
We give a topological version of a Bertini type theorem due to Abhyankar. A new definition of a branched covering is given. If the restriction $\pi_{V}: V \rightarrow Y$ of the natural projection $\pi: Y \times Z \rightarrow Y$ to a closed set $V \subset Y \times Z$ is a branched covering then, under certain assumptions, we can obtain generators of the fundamental group $\pi_{1}((Y \times Z) \backslash V)$.
\end{abstract}

Introduction. In his book [1, pp. 349-356], Abhyankar proves an interesting theorem called by him a "Bertini theorem" or a "Lefschetz theorem". The theorem expresses a topological fact in complex analytic geometry. The purpose of this paper is to restate this theorem and its proof in purely topological language. Our formulation reads as follows:

THEOREM 1. Let $Z$ be a connected topological manifold (without boundary) modeled on a real normed space $E$ of dimension at least 2 and let $Y$ be a simply connected and locally simply connected topological space. Suppose that $V$ is a closed subset of $Y \times Z$ and $\pi: Y \times Z \rightarrow Y$ denotes the natural projection. Assume that $\pi_{V}=\pi \mid V: V \rightarrow Y$ is a branched covering whose regular fibers are finite and whose singular set $\Delta=\Delta\left(\pi_{V}\right)$ does not disconnect $Y$ at any of its points. Set $X=(Y \times Z) \backslash V$ and $L=\{p\} \times Z$, where $p \in Y \backslash \Delta$. If there exists a continuous mapping $h: Y \rightarrow Z$ whose graph is contained in $X$, then the inclusion $i: L \backslash V \hookrightarrow X$ induces an epimorphism $i_{*}: \pi_{1}(L \backslash V) \rightarrow \pi_{1}(X)$.

We have adopted the following definition. For any topological spaces $Y$ and $Y^{*}$, a continuous, surjective mapping $\psi: Y^{*} \rightarrow Y$ is a (topological) branched covering if there exists a nowhere dense subset $\Delta$ of $Y$ such that $\psi \mid Y^{*} \backslash \psi^{-1}(\Delta): Y^{*} \backslash \psi^{-1}(\Delta) \rightarrow Y \backslash \Delta$ is a covering mapping. Notice that the singular set $\Delta$ of the branched covering $\psi$ is not unique, but there is a smallest $\Delta(\psi)$ among such sets. Clearly, $\Delta(\psi)$ is a closed subset of $Y$. Thus

1991 Mathematics Subject Classification: 57M12, 55Q52.

Key words and phrases: fundamental group, branched covering. 
the set $Y \backslash \Delta(\psi)$ of regular points is open. Topological branched coverings are studied in $[2]$.

The assumption in Theorem 1 that the set $\Delta$ does not disconnect $Y$ at any point of $\Delta$ means that for each $y \in \Delta$ and every connected neighborhood $U$ of $y$ in $Y$ there exists a smaller neighborhood $W$ of $y$ for which $W \backslash \Delta$ is connected.

\section{An equivalent version and a straightening property}

THEOREM 2 (cf. [1, (39.7)]). Suppose the assumptions of Theorem 1 are satisfied. Then, for every connected covering $\varphi: X^{*} \rightarrow X$ (i.e. $X^{*}$ is connected $)$, the set $\varphi^{-1}(L \backslash V)$ is connected.

Theorems 1 and 2 are equivalent due to the following simple but useful observation.

LEMma 1 (cf. [1, (39.3)]). Let a topological space $A$ as well as its subspace $B$ be connected and locally simply connected. Then the following are equivalent:

(1.1) the induced homomorphism $\pi_{1}(B) \rightarrow \pi_{1}(A)$ is an epimorphism,

(1.2) if $\eta: A^{*} \rightarrow A$ is any connected covering, then $\eta^{-1}(B)$ is connected,

(1.3) if $\eta: A^{*} \rightarrow A$ is the universal covering, then $\eta^{-1}(B)$ is connected.

While Abhyankar deals with the second version (Theorem 2), we prefer to prove Theorem 1 directly. We will use the following lemmas from Abhyankar's proof.

LEMMA 2 (cf. $\left[1,\left(39.2^{\prime}\right)\right]$ ). Let $\bar{B}$ be the closed unit ball centered at 0 in any real normed space and let $B$ and $S$ be the corresponding open ball and sphere. Assume that $l:[0,1] \rightarrow B$ is a continuous mapping such that $l(0)=0$. Then there exists a homeomorphism $\tau:[0,1] \times \bar{B} \rightarrow[0,1] \times \bar{B}$ such that $\beta \circ \tau=\beta, \tau \mid([0,1] \times S) \cup(\{0\} \times \bar{B})=\mathrm{id}$ and $\tau(\operatorname{graph} l)=[0,1] \times\{0\}$, where $\beta:[0,1] \times \bar{B} \rightarrow[0,1]$ is the natural projection.

Pro of. Take $(t, b) \in[0,1] \times \bar{B}$. If $b \neq l(t)$ then we can find a unique positive number $e(t, b)$ such that $\|e(t, b) b+(1-e(t, b)) l(t)\|=1$. The mapping $E:([0,1] \times \bar{B}) \backslash \operatorname{graph} l \ni(t, b) \mapsto e(t, b) \in[1, \infty)$ is locally bounded and its graph is closed, so it is continuous. We define

$$
\tau(t, b)= \begin{cases}(t, 0) & \text { if } b=l(t), \\ \left(t, b+\frac{1-e(t, b)}{e(t, b)} l(t)\right) & \text { if } b \neq l(t) .\end{cases}
$$

The inverse mapping $\tau^{-1}$ is

$$
\tau^{-1}(t, b)=(t, b+(1-\|b\|) l(t)) .
$$

Clearly, $\tau$ is the desired homeomorphism. 
Corollary. Let $B, \bar{B}$ and $S$ be as in Lemma 2. Assume that $l$ : $[a, b] \rightarrow B$, where $a \leq b$, is a continuous mapping and take $c \in[a, b]$. Then there exists a homeomorphism $\tau:[a, b] \times \bar{B} \rightarrow[a, b] \times \bar{B}$ such that $\beta \circ \tau=\beta, \tau \mid([a, b] \times S) \cup(\{c\} \times \bar{B})=$ id and $\tau(\operatorname{graph} l)=[a, b] \times\{l(c)\}$, where $\beta:[0,1] \times \bar{B} \rightarrow[0,1]$ is the natural projection.

LEMMA 3 (cf. [1, (39.2)]). Every manifold $M$ (without boundary) modeled on any real normed space $E$ has the following straightening property: For each set $J \subset[0,1] \times M$ such that the natural projection $\beta:[0,1] \times M \rightarrow[0,1]$ restricted to $J$ is a covering of finite degree, there exists a homeomorphism $\tau:[0,1] \times M \rightarrow[0,1] \times M$ which satisfies the following three conditions:

(2.1) $\beta \circ \tau=\beta$,

(2.2) $\tau \mid\{0\} \times M=\mathrm{id}$,

(2.3) $\tau(J)=[0,1] \times \alpha(J \cap(\{0\} \times M))$, where $\alpha:[0,1] \times M \rightarrow M$ is the natural projection.

Remark. Such a homeomorphism $\tau$ will be called a straightening homeomorphism. The segment $[0,1]$ can be replaced by any other segment $[a, b]$, where $a \leq b$.

Proof of Lemma 3 . Let $d$ denote the degree of the covering $\beta \mid J$. Notice that $J=\bigcup_{j=1}^{d}$ graph $l_{j}$, where $l_{j}:[0,1] \rightarrow M$ are continuous mappings with pairwise disjoint graphs. For each $t \in[0,1]$, choose a family $U_{1, t}, \ldots, U_{d, t}$ of neighborhoods of $l_{1}(t), \ldots, l_{d}(t)$, respectively, and a family of homeomorphisms $f_{j, t}$ from $\bar{U}_{j, t}$ onto the closed unit ball $\bar{B}$ in $E$ such that the sets $\bar{U}_{j, t}(j=1, \ldots, d)$ are pairwise disjoint and $f_{j, t}\left(U_{j, t}\right)=\operatorname{int} \bar{B}=B$. For every $t \in[0,1]$ there exists $\delta(t)>0$ such that $l_{j}\left(t^{\prime}\right) \in U_{j, t}$ for every $j=1, \ldots, d$ and $t^{\prime} \in[0,1] \cap(t-\delta(t), t+\delta(t))$. Set $V_{t}=[0,1] \cap(t-\delta(t), t+\delta(t))$. Take a finite set $\left\{\bar{t}_{1}, \ldots, \bar{t}_{n}\right\}$ such that $\left\{V_{\bar{t}_{i}}\right\}_{i=1}^{n}$ covers $[0,1]$ and a finite sequence $0=t_{0}<\ldots<t_{n}=1$ such that $I_{k}=\left[t_{k-1}, t_{k}\right] \subset V_{\bar{t}_{k}}$. Thus, $l_{j}\left(I_{k}\right) \subset U_{j, \bar{t}_{k}}$ for $k=1, \ldots, n$ and $j=1, \ldots, d$.

For every $k=1, \ldots, n$, we define a straightening homeomorphism $\tau_{k}$ : $I_{k} \times M \rightarrow I_{k} \times M$ using the Corollary on each $\bar{U}_{j, \bar{t}_{k}}(j=1, \ldots, d)$ and setting $\tau_{k}(t, m)=(t, m)$ for $m \in M \backslash \bigcup_{j=1}^{d} \bar{U}_{j, \bar{t}_{k}}$. Let $H_{k}=\left[0, t_{k}\right]$. For every $k=1, \ldots, n$, we can define a straightening homeomorphism $\zeta_{k}: H_{k} \times M \rightarrow$ $H_{k} \times M$ as follows:

1) $\zeta_{1}=\tau_{1}$,

2) if $\zeta_{k-1}$ is defined then $\zeta_{k}=\zeta_{k-1} \cup\left(\left(\mathrm{id} \times \xi_{k}\right) \circ \tau_{k}\right)$, where $\xi_{k}: M \ni$ $m \longmapsto\left(\alpha \circ \zeta_{k-1}\right)\left(t_{k-1}, m\right) \in M$ and $\alpha$ is the natural projection on $M$.

It is easy to check that $\tau=\zeta_{n}$ is the desired straightening homeomorphism. 
3. Proof of Theorem 1. Clearly, $X$ is a connected and locally simply connected space. Let $j: L \backslash V \hookrightarrow X \backslash(\Delta \times Z)$ and $k: X \backslash(\Delta \times Z) \hookrightarrow X$ be the inclusions. Then the proof falls naturally into two parts. phism.

Part 1. The mapping $j_{*}: \pi_{1}(L \backslash V) \rightarrow \pi_{1}(X \backslash(\Delta \times Z))$ is an epimor-

Let $u=(f, g):[0,1] \rightarrow X \backslash(\Delta \times Z)$ be any loop at $(p, h(p))$. We define a new loop $w=(\widetilde{f}, \widetilde{g}):[0,1] \rightarrow X \backslash(\Delta \times Z)$ by

$$
w(t)= \begin{cases}u(2 t) & \text { for } 0 \leq t \leq 1 / 2 \\ (f(2-2 t), h(f(2-2 t))) & \text { for } 1 / 2<t \leq 1\end{cases}
$$

Since $Y$ is simply connected, we have $[w]=[u]$. Define

$$
\begin{gathered}
A:[0,1] \ni t \mapsto(t, \widetilde{g}(t)) \in[0,1] \times Z, \\
\Omega:[0,1] \times Z \ni(t, z) \mapsto(\widetilde{f}(t), z) \in Y \times Z .
\end{gathered}
$$

The restriction $\beta \mid \Omega^{-1}(V)$ of the natural projection $\beta:[0,1] \times Z \rightarrow[0,1]$ is a covering of finite degree. By Lemma 3 , it has a straightening homeomorphism $\tau:[0,1] \times Z \rightarrow[0,1] \times Z$. Set $\widehat{t}=1 / 2-|t-1 / 2|$ and $\tau_{t}=(\alpha \circ \tau)(t, \cdot)$, where $\alpha:[0,1] \times Z \rightarrow Z$ is the natural projection. We can assume that $\tau_{t}=\tau_{\hat{t}}$ because $\widetilde{f}(t)=\widetilde{f}(\hat{t})$. The homotopy $H(t, s)=\left(\widetilde{f}(\widehat{t}(1-s)),\left(\tau_{\hat{t}(1-s)}^{-1} \circ \tau_{t} \circ \widetilde{g}\right)(t)\right)$ joins the loop $w=H(\cdot, 0)$ to the loop $H(\cdot, 1)$ whose image is in $L \backslash V$. Notice that $H(0, s)=H(1, s)=(p, h(p))$ for every $s \in[0,1]$. This implies that $[u]=[H(\cdot, 1)] \in j_{*}\left(\pi_{1}(L \backslash V)\right)$ and completes the proof of Part 1.

$\mathrm{P}$ art 2. The mapping $k_{*}: \pi_{1}(X \backslash(\Delta \times Z)) \rightarrow \pi_{1}(X)$ is an epimorphism.

Let $u=(f, g):[0,1] \rightarrow X$ be any loop at $(p, h(p))$. For every $t \in[0,1]$, there exists a neighborhood $U_{t} \times W_{t}$ of $u(t)$, where $U_{t}$ and $W_{t}$ are simply connected and $U_{t} \times W_{t} \subset X$. The family $V_{t}=u^{-1}\left(U_{t} \times W_{t}\right)(t \in[0,1])$ is an open covering of $[0,1]$. Choose a finite subcover $V_{k}(k=1, \ldots, n)$ and a sequence $0=t_{0}<\ldots<t_{n}=1$ such that $\left[t_{k-1}, t_{k}\right] \subset V_{k}(k=1, \ldots, n)$. Let $V_{k}=u^{-1}\left(U_{k} \times W_{k}\right)$ for every $k$.

The arc component $C_{k}$ of $U_{k} \cap U_{k+1}$ which contains $f\left(t_{k}\right)$ is open in $Y$. Since $\Delta \cap C_{k}$ is nowhere dense in $C_{k}$, there is a point $p_{k}$ in $C_{k} \backslash \Delta$ for $k=1, \ldots, n-1$. Let $p_{0}=p_{n}=p$. For every $k$, there exists an arc $v_{k}$ : $\left[t_{k-1}, t_{k}\right] \rightarrow U_{k} \backslash \Delta$ which joins $p_{k-1}$ to $p_{k}$, because $U_{k}$ is a connected and locally arcwise connected space with a closed, nowhere dense and nowhere disconnecting subspace $U_{k} \cap \Delta$ (see $[1,(14.5)]$ ). Similarly, there exist arcs $c_{k}:[0,1] \rightarrow C_{k} \backslash \Delta$ joining $c_{k}(0)=f\left(t_{k}\right)$ to $c_{k}(1)=p_{k}$ for $k=1, \ldots, n-1$. Let $c_{0}=c_{n}:[0,1] \ni t \mapsto p \in\left(U_{1} \cap U_{n}\right) \backslash \Delta$. For every $k$, there exists a homotopy $H_{k}:[0,1] \times\left[t_{k-1}, t_{k}\right] \rightarrow U_{k}$ joining $H_{k}(0, t)=f(t)$ to $H_{k}(1, t)=$ $v_{k}(t)$ which satisfies $H_{k}\left(s, t_{k-1}\right)=c_{k-1}(s)$ and $H_{k}\left(s, t_{k}\right)=c_{k}(s)$. Set $v=$ $\bigcup_{k=1}^{n} v_{k}:[0,1] \rightarrow Y \backslash \Delta$ and $H=\bigcup_{k=1}^{n} H_{k}:[0,1] \times[0,1] \rightarrow Y$. Then the 
homotopy $\widetilde{H}(s, t)=(H(s, t), g(t))$ joins the loop $\widetilde{H}(0, t)=u(t)$ to the loop $\widetilde{H}(1, t)=(v(t), g(t))$ whose image is in $X \backslash(\Delta \times Z)$. Since the image of $\widetilde{H}$ is in $X$ and $\widetilde{H}(s, 0)=\widetilde{H}(s, 1)=(p, h(p)),[u] \in k_{*}\left(\pi_{1}(X \backslash(\Delta \times Z))\right)$.

Acknowledgements. The author wishes to express his gratitude to W. Pawłucki for suggesting the problem. He also thanks W. Pawłucki and S. Łojasiewicz for stimulating comments.

\section{References}

[1] S. S. Abhyankar, Local Analytic Geometry, Academic Press, New York and London, 1964.

[2] A. Piękosz, Basic definitions and properties of topological branched coverings, to appear.

INSTITUTE OF MATHEMATICS

CRACOW UNIVERSITY OF TECHNOLOGY

WARSZAWSKA 24

31-155 KRAKÓW, POLAND

Reçu par la Rédaction le 11.4.1994 